\title{
How effective was Newfoundland \& Labrador's travel ban to prevent the spread of COVID-19? An agent-based analysis
}

\author{
Dionne M. Aleman, $\mathrm{PhD}^{1,2}$, Benjamin Z. Tham, MSc ${ }^{1}$, Sean J. Wagner, $\mathrm{PhD}^{3}$, Justin \\ Semelhago $^{1}$, Asghar Mohammadi, $\mathrm{MSc}^{4}$, Paul Price, $\mathrm{BSc}^{4}$, Randy Giffen, $\mathrm{MD}^{3}$, and Proton \\ Rahman, $\mathrm{MD}^{4,5}$ \\ ${ }^{1}$ Department of Mechanical \& Industrial Engineering, University of Toronto, Toronto, \\ Ontario, Canada \\ ${ }^{2}$ Institute for Health Policy, Management \& Evaluation, University of Toronto, Toronto, \\ Ontario, Canada \\ ${ }^{3}$ IBM, Toronto, Ontario, Canada \\ ${ }^{4}$ Faculty of Medicine, Memorial University, St. John's, Newfoundland \& Labrador, Canada \\ ${ }^{5}$ Eastern Health, St. John's, Newfoundland \& Labrador, Canada
}

February 23, 2021

\begin{abstract}
Background: To prevent the spread of COVID-19 in Newfoundland \& Labrador (NL), NL implemented a wide travel ban in May 2020. We estimate the effectiveness of this travel ban using a customized agent-based simulation (ABS).

Methods: We built an individual-level ABS to simulate the movements and behaviors of every member of the NL population, including arriving and departing travellers. The model considers individual properties (spatial location, age, comorbidities) and movements between environments, as well as age-based disease transmission with pre-symptomatic, symptomatic, and asymptomatic transmission rates. We examine low, medium, and high travel volume, traveller infection rates, and traveller quarantine compliance rates to determine the effect of travellers on COVID spread, and the ability of contact tracing to contain outbreaks.
\end{abstract}

Results: Infected travellers increased COVID cases by 2-52x (8-96x) times and peak hospitalizations by $2-49 \mathrm{x}(8-94 \mathrm{x})$, with (without) contact tracing. Although contact tracing was highly effective at reducing spread, it was insufficient to stop outbreaks caused by travellers in even the best-case scenario, and the likelihood of exceeding contact tracing capacity was a concern in most scenarios. Quarantine compliance had only a small impact on COVID spread; travel volume and infection rate drove spread.

Interpretation: NL's travel ban was likely a critically important intervention to prevent COVID spread. Even a small number of infected travellers can play a significant role in introducing new chains of transmission, resulting in exponential community spread and significant increases in hospitalizations, while outpacing contact tracing capabilities. With the presence of more transmissible variants, e.g., the UK variant, prevention of imported cases is even more critical. 


\section{Introduction}

On March 11, 2020, WHO declared SARS-CoV-2 (COVID) a pandemic with 118,000 cases largely from four countries [1]; four months later, there were 28 million cases globally and 1.8 million new cases per week. [2]. Empirical evidence indicates that travel restrictions reduce the spread of diseases in general [3, 4], and successful current real-life strategies for COVID include travel restriction as a crucial component. In China, travel restrictions imposed on January 23, 2020 resulted in a decrease of 515 to 39 travel-related cases in Wuhan just one week later [5], and an estimated three-day delay of COVID arrival [6]. Continued travel restrictions in New Zealand, part of a broad intervention package, led to one of the lowest case numbers and mortalities worldwide [7] and effective community elimination of COVID by June 2020 [8]. Similarly, Australia utilized both international and internal travel restrictions, controlling both initial and subsequent waves $[9]$.

Though seemingly effective at slowing COVID spread, the economic impact of travel restrictions is inconclusive, and mobility restrictions disproportionately impact poorer people [10] and workers in certain industries [11]. Further, travel restrictions may be legally controversial [12] and are only moderately effective unless implemented with other policies $[13,14]$. Thus, it is important to understand the potential benefit of travel restrictions for individual regions.

For the province of Newfoundland \& Labrador (NL) in Canada, strict restrictions on travel from out-of-province, implemented May 2020, have contributed to low case counts. NL has a large tourism economy, with annual non-resident travellers ( $\approx 533,000[15])$ exceeding the province's population $(\approx 520,000[16])$, as well as a large presence of rotational workers. Thus, there is considerable pressure to relax travel restrictions. While studies show that travel increases COVID spread, there is a reliance on models with "potentially inappropriate assumptions" [17]. We therefore use a granular agent-based simulation (ABS) of COVID spread in NL to estimate the effectiveness and appropriateness of NL's travel ban, finding that a small number of infected travellers can introduce new chains of transmission even with contact tracing, similar to documented case studies $[18,19]$. This finding is particularly important in the presence of new COVID variants of concern (VOCs) - particularly the UK variant, estimated to be $56 \%$ more transmissible than pre-existing variants [20] — as demonstrated by a recent outbreak at a long-term care home in Barrie, Ontario, where contact with an international traveller by a staff member quickly led to the infection of 127 residents and at least 32 deaths [21].

\section{Methods}

\subsection{The agent-based simulation model}

The ABS is adapted from morPOP (morLAB Pandemic Outbreak Planner), an ABS built to simulate the spread of pandemic influenza in the Greater Toronto Area, Ontario, Canada [22]. Specific adaptations for COVID include the delineation of pre-symptomatic (latent), symptomatic, and asymptomatic patient statuses. New features include travellers and contact tracing, while public transportation usage is removed as public transit is not heavily used in NL and little transit data was available. The population is constructed based on data from Statistics Canada's 2016 census [16] and provincial departments, and households are located in census subdivisions (CSDs) according to the census; household compositions (single person, two adults, two adults with one child, etc.) are similarly determined by CSD-level census data. 
morPOP models each individual in the population as a unique entity, allowing for individual behavioral, demographic, and health characteristics to be represented. Unique environments where people interact are represented: households, hospitals, schools, businesses, etc. Individuals have random community contacts in the CSDs where they live and work. Each individual has an infection status following the typical SIRD (susceptible, infectious, recovered, dead) model, with a latent asymptomatic infectious period after initial infection. During the simulation, individuals follow behavior patterns like being at home for a certain number of hours per day, going to work or school, observing physical distancing guidelines or not, and seeking medical care when infected. Visits to primary care physicians (assumed to be all in-person) and hospitals are captured, but use of advanced medical care (e.g., ventilators) is not.

At each location visited by an individual, there is interaction with other individuals at the same location (household members, fellow students, fellow employees, etc.), and an individual's chance of becoming infected is determined by contact with infected individuals in each location and the nature of that contact. For example, contact in a household may be much more likely to result in infection than contact with a customer at a business. Individuals' health statuses are updated each simulated day, and the probability that a susceptible person $j$ becomes infected on day $n$ is calculated by

$$
\begin{aligned}
E_{i j}^{n} & =\alpha_{i j}\left(t_{i j}^{n} b_{i j}^{n}+c_{i j}^{n}\right) & & \text { person } i-j \text { exposure } \\
Q_{i j}^{n} & =1-E_{i j}^{n} & & \operatorname{Pr}(j \text { not infected by } i) \\
\operatorname{Pr}_{j}^{n}(\mathrm{~S}, \mathrm{I}) & =1-\prod_{i \in \mathcal{I}} Q_{i j}^{n} & & \operatorname{Pr}(j \text { infected tomorrow })
\end{aligned}
$$

where $\alpha_{i j}$ is the Vancouver School of Economics (VSE) risk factor for the environment in which person $i$ and person $j$ have contact [23]; $t_{i j}^{n}$ is the time (in minutes) of contact between $i$ and $j$ on day $n ; b_{i j}^{n}$ is the rate of disease transmission between $i$ and $j$ per minute of contact; $c_{i j}^{n}$ is indirect transmission between $i$ and $j$; and $\mathcal{I}$ is the set of currently infected individuals.

Travellers arrive to CSDs either singly or in travel parties at a rate determined by monthly travel volumes, and each travel party (including a travel party of size one) forms its own household. The entire travel party is assigned to a workplace in the CSD if the visit purpose is business, or is "bubbled" with another household in the CSD if the visit purpose is to visit friends/family. For other visit reasons, the traveller (or travel party) only has random community contacts with residents. After a pre-determined length of stay, travellers leave the simulation. Infected travellers count towards the province's infections while they are in the province, but not after they leave.

Specific limitations of the current implementation of morPOP for NL-COVID, and whether they are likely to result in an under- or overestimation of infections, are in Table 1.

\subsection{Model parameters}

The main parameters for the ABS are shown in Table 2. Transmission rates are adapted from influenza per-minute age-based transmission rates [24]. Contacts in workplaces are adjusted by VSE Risk Factor [23, 24], and death rate is adjusted by age and comorbidity [25-27], where comorbidities are assigned to individuals by prevalence per CSD (publicly available Canadian Community Health Survey and Statistics Canada data). Hazard rates for identified comorbidities of interest (Table 3) 
Table 1: Limitations and potential bias of morPOP predictions

\begin{tabular}{lll}
\hline Limitation & Bias & Explanation \\
\hline $\begin{array}{l}\text { Travellers for business assigned to random } \\
\text { workplaces in their CSD }\end{array}$ & Under & $\begin{array}{l}\text { Rotational workers are likely to work } \\
\text { in the same workplaces (e.g., fish } \\
\text { processing plant), significantly } \\
\text { increasing the chance of infection for } \\
\text { resident workers }\end{array}$ \\
$\begin{array}{lll}\text { Individual travellers only visit/work in one CSD } \\
\text { Transmission rates do not differentiate ages 5-18 }\end{array}$ & Under & $\begin{array}{l}\text { Opportunity for disease transmission } \\
\text { in multiple communities is not present }\end{array}$ \\
$\begin{array}{l}\text { Although still uncertain, it is believed } \\
\text { that younger children are less } \\
\text { contagious than older children }\end{array}$ \\
$\begin{array}{l}\text { Institutional facilities (long-term care homes, } \\
\text { prisons) are not modelled }\end{array}$ & Under & $\begin{array}{l}\text { Disease may spread rapidly in these } \\
\text { environments }\end{array}$ \\
$\begin{array}{l}\text { Transmission within hospitals not modelled } \\
\text { Specific nature of workplace contact (indoor, } \\
\text { outdoor, isolated offices/cubicles, etc.) not } \\
\text { modelled }\end{array}$ & Under & $\begin{array}{l}\text { Disease may spread in hospitals } \\
\text { All contacts and transmission within a } \\
\text { workplace are assumed to happen like }\end{array}$ \\
\hline
\end{tabular}

are applied to both death and hospitalization probabilities. Demographic parameters for individuals and households are based on 2016 NL census data [16]. Workplace, home-work commutes, hospital, and Regional Health Authority parameters are from NL-specific data. As the purpose of this study is to examine the effectiveness of NL's travel ban, transmission rates in non-household environments are scaled down by $80 \%$ to reflect protective behaviors (social distancing, masks) assumed to be commonplace during the first months of the pandemic.

All traveller parameters (Figure 1) are determined from an exit survey conducted by the Government of Newfoundland and Labrador Department of Tourism, Culture, Industry and Innovation [29]. This exit survey indicates traveller destinations by economic zone, which were mapped to CSDs.

\subsection{Travel scenarios}

To assess the impact of the travel ban, we compare a baseline scenario of the travel ban in place with low, medium, and high values of travel volumes, traveller infection rates $(\theta)$, and traveller quarantine compliance rates (Table 4). The impact of contact tracing on each scenario is examined by running each scenario with and without contact tracing, for a total of 56 scenarios.

A percentage of travellers $(50 \%, 75 \%, 80 \%)$ immediately quarantine upon arrival, meaning that they only have contacts within their household (travel party) for the duration of the quarantine. Of the travellers who did not quarantine, infected individuals will choose to begin quarantine upon symptom onset with a certain probability $(25 \%, 50 \%, 80 \%)$. These lower probabilities reflect the intuition that travellers who have already chosen to not follow quarantine rules are likely incentivized to continue not following those rules when symptoms appear due to the nature of their travel (strict timelines, work/family pressures) and demonstrated lack of adherence to quarantine 
Table 2: Model parameters

\begin{tabular}{|c|c|}
\hline Parameter & Value \\
\hline \multicolumn{2}{|l|}{ Population } \\
\hline Initial number of unknown infections in NL population & 5 \\
\hline Duration of self-isolation & 14 days \\
\hline Probability that a resident self-isolates upon symptom onset & $90 \%$ \\
\hline $\begin{array}{l}\text { Probability that an entire household self-isolates when one member becomes } \\
\text { symptomatic }\end{array}$ & $90 \%$ \\
\hline $\begin{array}{l}\text { Number of days after symptom onset that a person chooses to alter behavior, } \\
\text { with probabilities }\end{array}$ & $0(80 \%), 1(10 \%), 2(10 \%)$ \\
\hline Household bubble size & 2 \\
\hline Daily age-based contacts outside home/work/school & S1 Dataset, Canada [28] \\
\hline \multicolumn{2}{|l|}{ Travellers } \\
\hline Total annual non-resident travellers in a typical year (2018) & $533,507[15]$ \\
\hline $\begin{array}{l}\text { Monthly non-resident travellers to each census subdivision (matched to economic } \\
\text { zones) }\end{array}$ & Table $10[29]$ \\
\hline $\begin{array}{l}\text { Travel party size, assumed to be of size 1-4 individuals and tuned to match } \\
\text { known means }\end{array}$ & $\begin{array}{l}1(31 \%), 2(63 \%), 3(2 \%), 4 \\
(4 \%)(\text { Table } 5[29])\end{array}$ \\
\hline $\begin{array}{l}\text { Percent of travel parties who are assigned to a workplace (travel for business or } \\
\text { conference) }\end{array}$ & $29 \%$ (Table $3[29]$ ) \\
\hline $\begin{array}{l}\text { Percent of travel parties who bubble with one household (travel for visiting } \\
\text { relatives or other) }\end{array}$ & $36 \%$ (Table $3[29]$ ) \\
\hline $\begin{array}{l}\text { Length of stay (days) of non-resident parties, tuned so that the } 32.1 \text {-day average } \\
\text { stay for business travellers by auto is } 4 \text { standard deviations from the mean }\end{array}$ & $\begin{array}{l}\text { Normal }(10.8,5.3) \text { (Table } 6 \\
[29])\end{array}$ \\
\hline $\begin{array}{l}\text { Number of days already been infected upon arrival for infected travellers } \\
\text { (maximum possible is } 5 \text { days latent }+10 \text { days symptomatic) }\end{array}$ & $0-15$ days \\
\hline \multicolumn{2}{|l|}{ Contact tracing } \\
\hline Daily contact tracing call capability & 1932 calls/day \\
\hline $\begin{array}{l}\text { Days after being infected that contact tracing begins (includes latent period, } \\
\text { days with symptoms before getting tested, and turnaround time for test result) }\end{array}$ & 9 days \\
\hline Number of days before symptom onset to contact trace & 2 days \\
\hline Percent of non-medical-care seeking individuals that are contact-traced & $50 \%$ \\
\hline Percent of known contacts that are successfully found and contacted & $95 \%$ \\
\hline Percent of contacts outside home/work/school that an individual remembers & $90 \%$ \\
\hline $\begin{array}{l}\text { Number of employees for workplace to be small enough to be closed due to a } \\
\text { single infection }\end{array}$ & 10 \\
\hline \multicolumn{2}{|l|}{ Disease } \\
\hline Per-minute disease transmission rates between age groups & Table A1 [24] \\
\hline Percent of asymptomatic cases & $42.5 \%[30]$ \\
\hline $\begin{array}{l}\text { Contagiousness of pre-symptomatic and asymptomatic cases compared to } \\
\text { symptomatic }\end{array}$ & $25 \%[31]$ \\
\hline Latent period & 5 days \\
\hline Symptomatic period & $6-10$ days \\
\hline
\end{tabular}


Table 3: Hazard rates by comorbidity for death and hospitalization probabilities

Comorbidity

Cancer

Chronic obstructive pulmonary disease (COPD)

Diabetes

High blood pressure

Ischemic heart disease (IHD)

Obesity

Asthma
Hazard rate

2.05

1.90

1.90

1.58

1.40

1.40

1.25

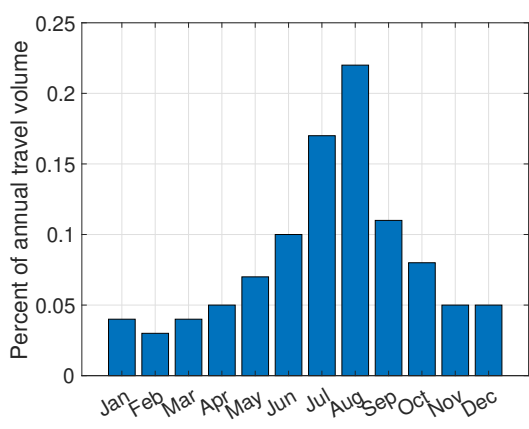

(a) Monthly travel volume

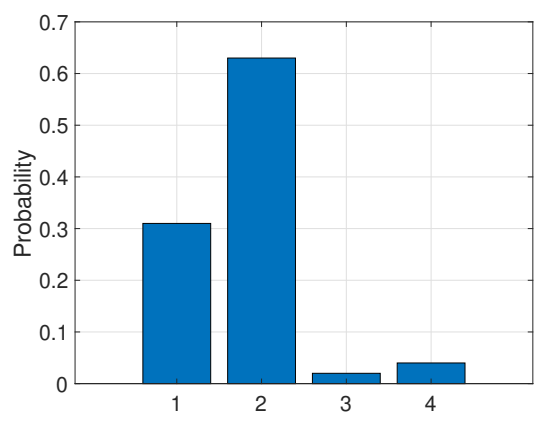

(b) Travel party size

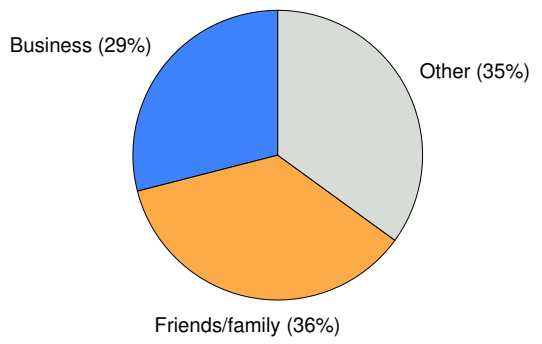

(c) Travel reason

Figure 1: Traveller arrival details 
Table 4: Low, medium, and high values in traveller scenarios

\begin{tabular}{lccc}
\hline & Travel volume* & $\begin{array}{c}\text { Traveller infection } \\
\text { rate }(\theta)\end{array}$ & $\begin{array}{c}\text { Traveller quarantine compliance rates } \\
\text { (immediately / after symptom onset) }\end{array}$ \\
\hline Baseline & $0 \%$ (travel ban) & - & - \\
Low & $24 \%$ & $0.03 \%$ & $50 \% / 25 \%$ \\
Medium & $50 \%$ & $0.1 \%$ & $75 \% / 50 \%$ \\
High & $100 \%$ & $1 \%$ & $80 \% / 80 \%$ \\
\hline
\end{tabular}

* Travel volume is in terms of non-resident travel volume; $24 \%$ non-resident travel volume is approximately $10 \%$ total travel volume (non-resident + resident).

rules.

Reasonable low $(0.03 \%)$, medium $(0.1 \%)$, and high $(1 \%)$ values for $\theta$ were chosen in consultation with NL Public Health, noting that a $1 \%$ infection rate was calculated at Toronto Pearson International Airport from September to November 2020 [32]. The medium (50\%) and high (100\%) travel volumes were similarly estimated at the time the travel ban was implemented. After the summer, St. John's International Airport in NL reported that travel volumes were in fact $10 \%$ of typical volumes. As the available travel volume data [29] is for non-resident travel only, $10 \%$ of total travel volumes is approximately $24 \%$ non-resident travel volumes, and thus, $24 \%$ travel volume is examined as the low travel volume scenario.

The scenarios start on May 1 and simulate 100 days. An initial undetected five cases are present at the start of the simulation. Although NL had no detected cases on May 1, without full-scale population testing, it is reasonable to assume that there are a small number of existing infections that are asymptomatic or have sufficiently mild symptoms so as not to require hospitalization. Households are engaged a "double bubble", meaning that households have close contact with one other household, in accordance with NL's de-escalation protocol during the time period.

\subsection{Model implementation}

Unlike most ABSs that struggle to capture large populations or that require lengthy computation times and memory, morPOP is written in $\mathrm{C}++$ for computational speed and parallelization. Using a number of computational techniques specifically designed to speed up run times, morPOP simulated a single 100-day outbreak on the NL population of $\approx 520,000$ agents in on average $1.8 \mathrm{~min}$ without travellers or contact tracing; 5min with travellers; and $8.3 \mathrm{~min}$ with travellers and contact tracing. The difference in time is primarily due to dynamic memory allocation required by travellers and contact tracing. The model was parallelized with one simulation per processor and implemented on high-performance computing infrastructure provided by the Center for Health Informatics and Analytics (CHIA) at Memorial University. The specific infrastructure used was three Linux nodes, each with 32 cores (64 threads) and 256 GB RAM, allowing for 192 simulations to be run simultaneously. Thus, a run of 500 simulations requires $\approx 5-25 \mathrm{~min}$, depending on the presence of travellers and contact tracing. 
Table 5: Magnitude increase over baseline (travel ban) scenario, without contact tracing. Ranges reflect differences due to traveller quarantine compliance.

\begin{tabular}{l|ccc|ccc}
\hline \multirow{2}{*}{ Travel volume } & \multicolumn{3}{|c|}{ Mean total cases } & \multicolumn{3}{|c}{ Mean peak hospitalizations } \\
& $\theta=0.03 \%$ & $\theta=0.1 \%$ & $\theta=1 \%$ & $\theta=0.03 \%$ & $\theta=0.1 \%$ & $\theta=1 \%$ \\
\hline $100 \%$ & $14-18 \mathrm{x}$ & $20-24 \mathrm{x}$ & $80-96 \mathrm{x}$ & $14-18 \mathrm{x}$ & $20-25 \mathrm{x}$ & $77-94 \mathrm{x}$ \\
$50 \%$ & $10-12 \mathrm{x}$ & $18-19 \mathrm{x}$ & $52-60 \mathrm{x}$ & $10-12 \mathrm{x}$ & $17-20 \mathrm{x}$ & $53-64 \mathrm{x}$ \\
$24 \%$ & $8-11 \mathrm{x}$ & $12-14 \mathrm{x}$ & $28-33 \mathrm{x}$ & $8-10 \mathrm{x}$ & $12-14 \mathrm{x}$ & $29-35 \mathrm{x}$ \\
\hline
\end{tabular}

\section{Results}

Five hundred simulations were performed for each scenario. To assess the impact of contact tracing, we first analyze scenarios without contact tracing, followed by the same scenarios with contact tracing.

\subsection{Without contact tracing}

In the baseline (travel ban) scenario, the number of infections reaches 0 in late June, while all other scenarios show exponential increase (Figure 2, top). Travellers' quarantine compliance does not have a large impact on community disease spread; once infected travellers initiate community disease spread, exponential growth of infections begins. Travel volumes, however, significantly impact disease spread, with $1 \%$ infected travellers yielding such high numbers of cases that the figure had to be plotted on a different scale. Table 5 indicates the magnitude increase in mean total cases and hospitalizations over baseline for each scenario, again illustrating that quarantine compliance impacts total cases far less than travel volume and infection rate.

Because mean and confidence interval plots like those in active case plots of Figure 2 (top) obscure simulation outcomes that are much larger than the mean (e.g., super-spreader events), simulation outcomes are better illustrated by examining the probability that metrics exceed thresholds. The other rows in Figure 2 illustrate the probability that the peak number of cases, total cases, deaths, and hospitalizations exceeds the threshold in the $x$-axis. In all metrics, for a fixed rate of infection, the probability of increasingly worse outcomes increases as the travel volume increases and rate of compliance declines. As the rate of infection increases, the outcomes all worsen dramatically, particularly as the rate of infection grows to $1 \%$.

\subsection{With contact tracing}

Contact tracing is highly effective at reducing disease spread (Figure 3), but the predictions of daily active COVID cases still exhibit exponential growth even in the best-case scenario of $24 \%$ travel volume, $\theta=0.03 \%$, and high quarantine compliance. The number of cases is still determined much more by travel volume and traveller infection rate rather than compliance, and contact tracing is insufficient to stop community spread from travellers (Table 6), which also results in significantly more hospitalizations than with the travel ban in place.

Contact tracing reduces the mean total number of cases by $45.5 \%$ in the baseline scenario, and is effective in the presence of travellers (though still unable to stop exponential growth), but 

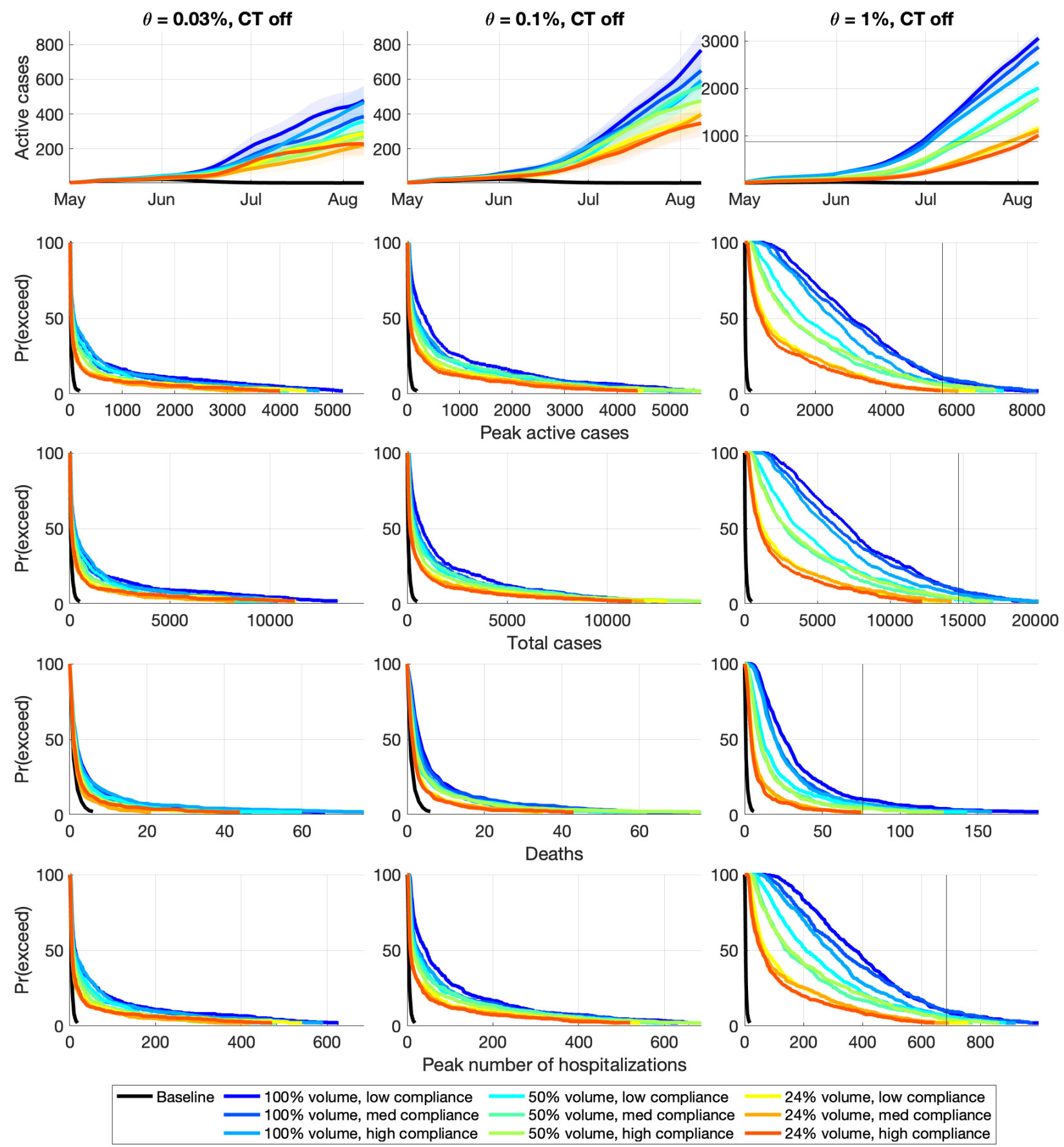

Figure 2: Simulation outcomes without contract tracing (CT). 95\% confidence intervals are shown, and the mean is bolded. $\theta$ is the traveller infection rate. Note that $\theta=1 \%$ is on a different scale; the horizontal line at $\approx 800$ cases in $\theta=1 \%$ active cases (top) indicates the upper limit of the figures for $\theta=0.03 \%$ and $0.1 \%$, while the vertical lines in other rows indicate the maximum $x$-axis value for $\theta=0.03 \%$ and $0.1 \%$. 

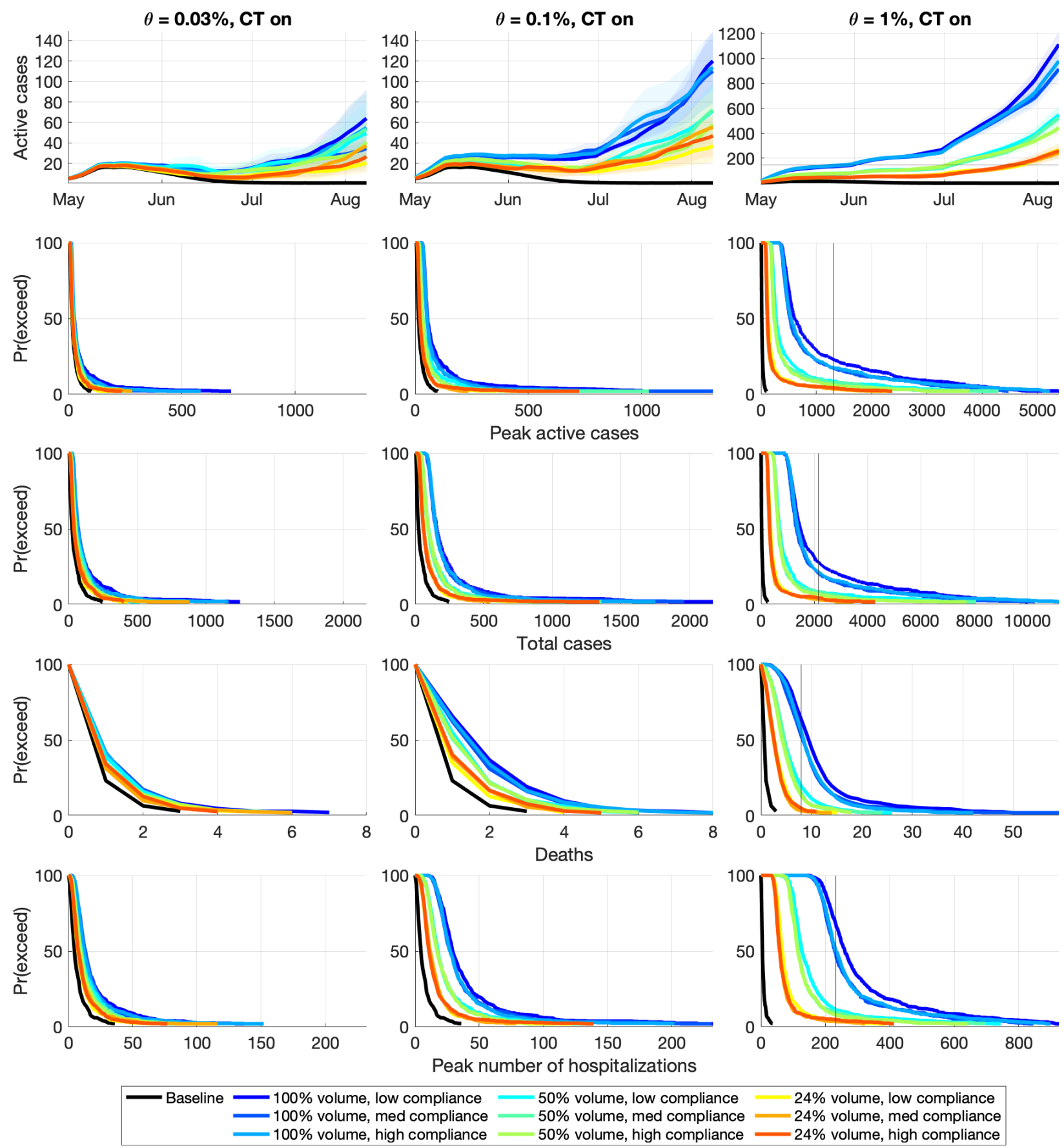

Figure 3: Simulation outcomes with contract tracing (CT). 95\% confidence intervals are shown, and the mean is bolded. $\theta$ is the traveller infection rate. Note that $\theta=1 \%$ is on a different scale; the horizontal line at $\approx 140$ cases in $\theta=1 \%$ active cases (top) indicates the upper limit of the figures for $\theta=0.03 \%$ and $0.1 \%$, while the vertical lines in other rows indicate the maximum $x$-axis value for $\theta=0.03 \%$ and $0.1 \%$. 
Table 6: Magnitude increase over baseline (travel ban) scenario, with contact tracing. Ranges reflect differences due to traveller quarantine compliance.

\begin{tabular}{l|ccc|ccc}
\hline & \multicolumn{3}{|c|}{ Mean total cases } & \multicolumn{3}{c}{ Mean peak hospitalizations } \\
Travel volume & $\theta=0.03 \%$ & $\theta=0.1 \%$ & $\theta=1 \%$ & $\theta=0.03 \%$ & $\theta=0.1 \%$ & $\theta=1 \%$ \\
\hline $100 \%$ & $3-4 \mathrm{x}$ & $6-7 \mathrm{x}$ & $46-52 \mathrm{x}$ & $3-4 \mathrm{x}$ & $6-7 \mathrm{x}$ & $43-49 \mathrm{x}$ \\
$50 \%$ & $3-4 \mathrm{x}$ & $4 \mathrm{x}$ & $23-26 \mathrm{x}$ & $3 \mathrm{x}$ & $4 \mathrm{x}$ & $22-25 \mathrm{x}$ \\
$24 \%$ & $2-3 \mathrm{x}$ & $3-4 \mathrm{x}$ & $12-13 \mathrm{x}$ & $2 \mathrm{x}$ & $3 \mathrm{x}$ & $12-13 \mathrm{x}$ \\
\hline
\end{tabular}

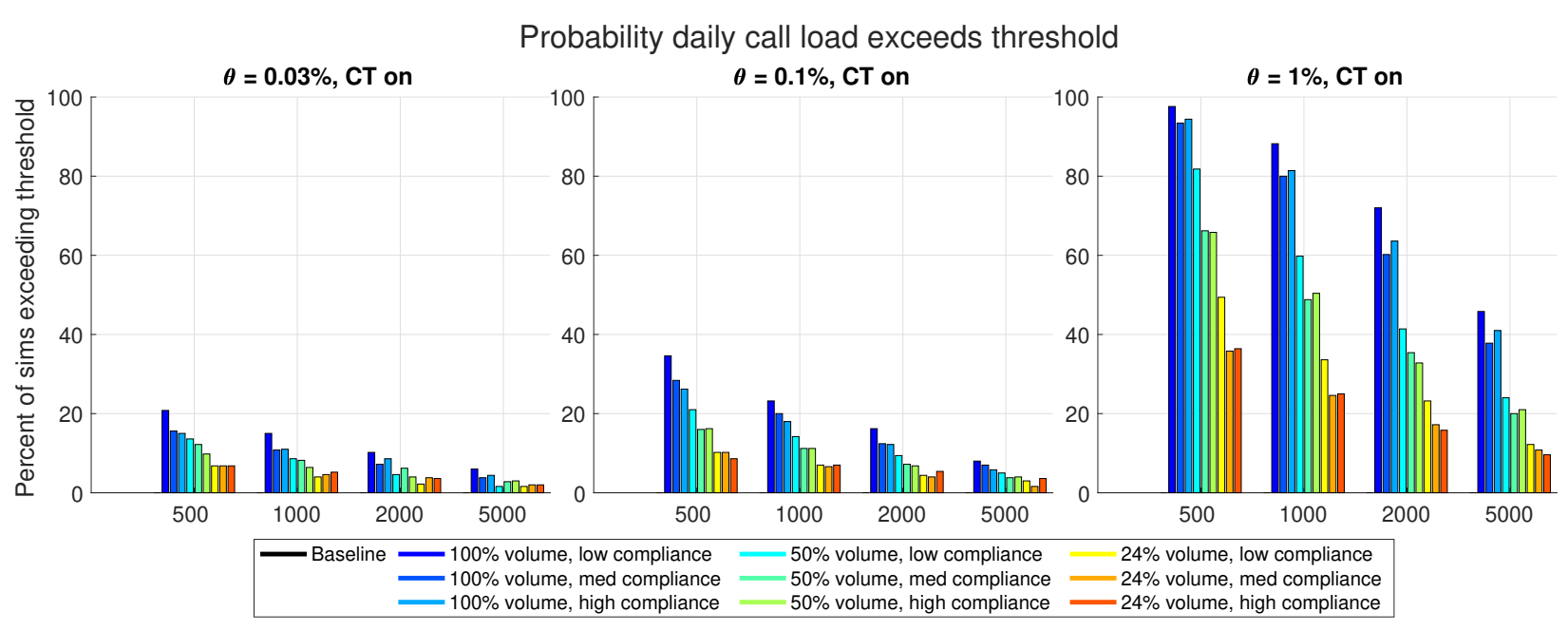

Figure 4: Contact tracing daily call load

effectiveness decreases as the rate of infected travellers increases. At $\theta=0.03 \%$ and $\theta=0.1 \%$, contact tracing reduces the mean total number of cases by $81.4-89.6 \%$, and by $67.6-79.3 \%$ for $\theta=1 \%$.

The daily call capacity for NL is 1932 calls, which has about a $10 \%$ and $16 \%$ chance of being exceeded in the worst-case travel volume and compliance scenarios for $\theta=0.03 \%$ and $\theta=0.1 \%$, respectively (Figure 4). For $\theta=1 \%$, the best-case travel volume scenario has call overload around $15-23 \%$ likelihood; the other scenarios have a $32-72 \%$ chance of call overload, and a $21-45 \%$ chance of even exceeding 5000 calls per day.

\section{Interpretations}

If the existing number of cases in NL is very small as was believed at the time of the travel ban, even a very small number of infected travellers had the potential to dramatically worsen the pandemic even with high levels of traveller quarantine compliance. However, the mere act of travel, especially by air (which comprises $84 \%$ of NL's non-resident travel [29]), increases exposure to COVID, and travellers may therefore have a higher risk of infection than the general population of their province or country of origin. Contact tracing has a tremendous ability to reduce the number of cases in the 
community, but even the best-case scenario of travellers begins exponential growth before the end of the 100-day simulation period. In all scenarios with travellers, both the mean total cases and peak number of hospitalizations were orders of magnitude larger than with the travel ban in place. The likelihood of exceeding contact tracing capacity is a concern even for lower rates of traveller infection at full travel volume, and capacity is very likely to be exceeded at a $1 \%$ rate of infected travellers.

Travel restrictions may be lessened with screening, however, allowing more travel means an increase in imported cases given the potential for false negatives [33-35], especially for asymptomatic and pre-symptomatic cases that may comprise close to half of infected travellers [36]. As demonstrated, the potential for a failure to contain COVID is high even with few infected travellers in the community, so any policy to reduce quarantine requirements must be carefully investigated with detailed knowledge of travellers' points of origin and likely infection rates; however, such information is difficult to ascertain given potentially convoluted travel paths.

Thus, the morPOP simulation model indicates that the travel ban provided significant protection to the NL population. With more transmissible VOCs recently discovered, the importance of preventing imported cases from travellers is even more critical than the model results suggest.

\section{References}

[1] World Health Organization. WHO Director-General's opening remarks at the media briefing on COVID-19 - 11 March 2020. WHO; 2020. Accessed January 23, 2021. https://www.who.int/dg/speeches/detail/ who-director-general-s-opening-remarks-at-the-media-briefing-on-covid-19---11-march-2020.

[2] World Health Organization. Coronavirus disease (COVID-19): weekly epidemiological update [Technical documents]. World Health Organization; 2020. Accessed January 23, 2021. https://www.who.int/docs/default-source/coronaviruse/situation-reports/ 20200914-weekly-epi-update-5.pdf.

[3] Brownstein JS, Wolfe CJ, Mandl KD. Empirical evidence for the effect of airline travel on inter-regional influenza spread in the United States. PLOS Med. 2006;3(10):e401.

[4] Bell DM, WHO Working Group on Prevention of International and Community Transmission of SARS. Public health interventions and SARS spread, 2003. Emerging Infectious Diseases. 2004;10(11):1900.

[5] Tian HY, Liu YH, Li YD, Wu CH, Chen B, Kraemer MUG, et al. An investigation of transmission control measures during the first 50 days of the COVID-19 epidemic in China. Science. 2020;368(6491):638-642.

[6] Kraemer MUG, Yang CH, Gutierrez B, Wu CH, Klein B, Pigott DM, et al. The effect of human mobility and control measures on the COVID-19 epidemic in China. Science. 2020;368(6490):493-497.

[7] Jefferies S, French N, Gilkison C, Graham G, Hope V, Marshall J, et al. COVID-19 in New Zealand and the impact of the national response: a descriptive epidemiological study. The Lancet Public Health. 2020;5(11):e612-e623. 
[8] Baker MG, Wilson N, Anglemyer A. Successful elimination of COVID-19 transmission in New Zealand. New England Journal of Medicine. 2020;383(8):e56.

[9] A A, M M, D RA, J T, E M. Delaying the COVID-19 epidemic in Australia: evaluating the effectiveness of international travel bans. Aust N Z J Public Health. 2020;44(4):257-259.

[10] Bonaccorsi G, Pierri F, Cinelli M, Flori A, Galeazzi A, Porcelli F, et al. Economic and social consequences of human mobility restrictions under COVID-19. Proceedings of the National Academy of Sciences. 2020;117(27):15530-15535.

[11] Nicola M, Alsafi Z, Sohrabi C, Kerwan A, Al-Jabir A, Iosifidis C, et al. The socio-economic implications of the coronavirus pandemic (COVID-19): A review. International Journal of Surgery (London, England). 2020;78:185.

[12] Meier BM, Habibi R, Yang YT. Travel restrictions violate international law. Science. 2020;367(6485):1436.

[13] Chinazzi M, Davis JT, Ajelli M, Gioannini C, Litvinova M, Merler S, et al. The effect of travel restrictions on the spread of the 2019 novel coronavirus (COVID-19) outbreak. Science. 2020;368(6489):395-400.

[14] Epstein JM, Goedecke DM, Yu F, Morris RJ, Wagener DK, Bobashev GV. Controlling pandemic flu: the value of international air travel restrictions. PLOS ONE. 2007;2(5):e401.

[15] Government of Newfoundland and Labrador Department of Tourism, Culture, Industry and Innovation. Newfoundland and Labrador Provincial Tourism Performance 2018; 2018. Accessed June 16, 2020. https://www.gov.nl.ca/tcii/files/Annual_Performance_Report_2018_ Final_June_-2019.pdf.

[16] Statistics Canada. 2016 Census; 2016.

[17] Burns J, Movsisyan A, Stratil JM, Coenen M, Emmert-Fees KMF, Geffert K, et al. Travelrelated control measures to contain the COVID-19 pandemic: a rapid review. Cochrane Database of Systematic Reviews. 2020;9:CD013717.

[18] Böhmer MM, Buchholz U, Corman VM, Hoch M, Katz K, Marosevic DV, et al. Investigation of a COVID-19 outbreak in Germany resulting from a single travel-associated primary case: a case series. The Lancet Infectious Diseases. 2020;20(8):920-928.

[19] Hâncean MG, Perc M, Lerner J. Early spread of COVID-19 in Romania: imported cases from Italy and human-to-human transmission networks. Royal Society Open Science. 2020;7(7):200780.

[20] Davies NG, Barnard RC, Jarvis CI, Kucharski AJ, Munday J, Pearson CAB, et al. Estimated transmissibility and severity of novel SARS-CoV-2 Variant of Concern 202012/01 in England. medRxiv. 2020;Available from: https://www.medrxiv.org/content/early/2020/ $12 / 26 / 2020 \cdot 12 \cdot 24.20248822$.

[21] Morris S. U.K. variant confirmed at Barrie, Ont., care home where 32 have died. CTV News; 2021. Accessed January 23, 2021. https://barrie.ctvnews.ca/ u-k-variant-confirmed-at-barrie-ont-care-home-where-32-have-died-1.5279379. 
[22] Aleman DM, Wibisono TG, Schwartz B. A nonhomogeneous agent-based simulation approach to modeling the spread of disease in a pandemic outbreak. INFORMS Journal on Applied Analytics. 2011;41(3):301-315.

[23] Vancouver School of Economics. Employment and COVID-19 Transmission Risks; 2020. Accessed July 8, 2020. https://lmic-cimt.shinyapps.io/vse_lmic_risk_analysis/.

[24] Haber MJ, Shay DK, Davis XM, Patel R, Jin XP, Weintraub E, et al. Effectiveness of interventions to reduce contact rates during a simulated influenza pandemic. Emerging Infectious Diseases. 2007;13(4):581.

[25] Coggon D, Croft P, Cullinan P, Williams A. Assessment of workers' personal vulnerability to COVID-19 using 'covid-age'. Occupational Medicine. 2020;70(10):461-464.

[26] Guan WJ, Liang WH, Zhao Y, Liang HR, Chen ZS, Li YM, et al. Comorbidity and its impact on 1590 patients with COVID-19 in China: a nationwide analysis. European Respiratory Journal. 2020;55(5).

[27] Docherty AB, Harrison EM, Green CA, Hardwick HE, Pius R, Norman L, et al. Features of 16,749 hospitalised UK patients with COVID-19 using the ISARIC WHO Clinical Characterisation Protocol. medRxiv. 2020;Available from: https://www.medrxiv.org/content/ early/2020/04/28/2020.04.23.20076042.

[28] Prem K, Cook AR, Jit M. Projecting social contact matrices in 152 countries using contact surveys and demographic data. PLOS Computational Biology. 2017;13(9):e1005697.

[29] Government of Newfoundland and Labrador Department of Tourism, Culture, Industry and Innovation. 2016 Exit Survey - Results Highlights; 2018. Accessed June 16, 2020. https://www.gov.nl.ca/tcii/files/2016_Exit_Survey_Highlights_Report_ FINAL_REVISED_June_2018.pdf.

[30] Lavezzo E, Franchin E, Ciavarella C, Cuomo-Dannenburg G, Barzon L, Vecchio CD, et al. Suppression of a SARS-CoV-2 outbreak in the Italian municipality of Vo. Nature. 2020;584:425429.

[31] Alberta Health Services. COVID-19 Scientific Advisory Group Rapid Response Report; 2020. Accessed July 8, 2020. https://www.albertahealthservices.ca/assets/info/ppih/ if-ppih-covid-19-rapid-response-asymptomatic-transmission.pdf.

[32] McMaster HealthLabs. McMaster HealthLabs Releases Interim Report on COVID19 Study of Arriving International Travellers; 2020. Accessed December 1, 2020. https://www. mcmasterhealthlabs.ca/pdf/MHL\%20Border\%20Study\%20Interim\% 20Results\%20News\%20Release_20201117_vF_EN.pdf.

[33] West CP, Montori VM, Sampathkumar P; Elsevier. COVID-19 testing: the threat of falsenegative results. Mayo Clinic Proceedings. 2020;95(6):1127-1129.

[34] Winichakoon P, Chaiwarith R, Liwsrisakun C, Salee P, Goonna A, Limsukon A, et al. Negative nasopharyngeal and oropharyngeal swabs do not rule out COVID-19. Journal of Clinical Microbiology. 2020;58(5). 
medRxiv preprint doi: https://doi.org/10.1101/2021.02.05.21251157; this version posted February 23, 2021. The copyright holder for this preprint

(which was not certified by peer review) is the author/funder, who has granted medRxiv a license to display the preprint in perpetuity.

It is made available under a CC-BY-NC-ND 4.0 International license .

[35] Xiao AT, Tong YX, Zhang S. False-negative of RT-PCR and prolonged nucleic acid conversion in COVID-19: Rather than recurrence. Journal of Medical Virology. 2020;92(10):1755-1756.

[36] Wong J, Abdul Aziz ABZ, Chaw L, Mahamud A, Griffith MM, Lo YR, et al. High proportion of asymptomatic and presymptomatic COVID-19 infections in air passengers to Brunei. Journal of Travel Medicine. 2020;27(5):taaa066. 